\title{
Rétrospective et perspective
}

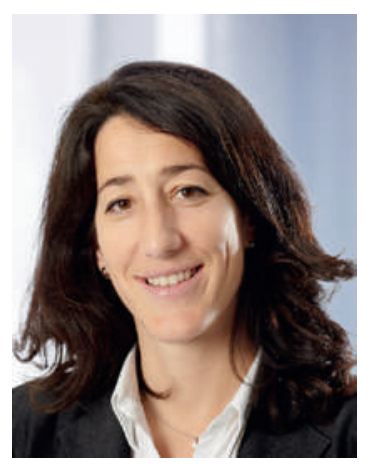

La période des fêtes de fin d'année est pour chacun d'entre nous le temps d'un retour sur l'année écoulée mais également celui de la réflexion sur nos attentes et nos espoirs pour les mois à venir.

A l'heure du bilan donc on se demandera si on a été fidèle à la ligne que l'on avait choisi de suivre et si on a vraiment engagé tous les moyens nécessaires pour atteindre les objectifs qu'on s'était fixés. On repensera aux aléas de la vie qui nous ont peut-être fait dévier de notre route nous rendant la tâche plus ardue ou plus douce.

Pour le Secrétariat général, cette année 2012 n'a pas été des plus simples. Elle a été synonyme de changements, d'absences, de départs, mais aussi de renouveau, de projets, de visions. Le bilan est positif. Aux premières étapes de restructurations et à l'introduction des premiers nouveaux processus de gestion se sont ajoutés notamment le changement de quelques membres du Comité central et les nouvelles affectations des ressorts. Pour ces derniers, l'année 2012 a vu la réalisation de certains projets ou le renvoi à l'année à venir d'autres d'entre eux. Pour moi, l'année 2012 a été celle d'un pilotage à distance, épaulée par une équipe fantastique qui a su avec engagement poursuivre l'activité et les efforts engagés. Je ne peux ici que remercier les collaboratrices et les collaborateurs du Secrétariat général qui ont rendu ceci pos-

\section{En devenant plus performants et en optimisant les dépenses, le Secrétariat général pourra encore mieux servir les membres de la FMH et représenter cette dernière.}

sible. A tous ceux qui s'interrogent sur la nécessité du travail du Secrétariat général et sur les efforts mis en œuvre pour améliorer ses structures et ses modes de fonctionnement, j'aimerais répondre que c'est en devenant plus performants et en optimisant nos dépenses que l'on pourra encore mieux servir les membres de la FMH et représenter cette dernière.
Enfin pour la FMH, l'année 2012 aura assurément été celle d'une présence politique et médiatique forte et de sa reconnaissance confirmée comme acteur incontournable du monde de la santé.

A l'heure des espoirs et des envies, on repensera aux vœux de Noël, à l'espérance d'un monde de paix, de réconciliation et d'harmonie. On se fixera de nouveaux objectifs à atteindre ensemble. On choisira de poursuivre ceux qui nous ont guidés cette dernière année et qui ne sont pas encore achevés. Mais en tous les cas au sein du Secrétariat général, conscients des enjeux majeurs que sont ceux du monde de la santé d'aujourd'hui et de demain, ainsi que des mutations qu'il va devoir vivre pour répondre aux attentes, nous allons continuer notre mission. Nous mènerons aussi loin que possible notre réforme interne et notre professionnalisation. Nous continuerons de servir au mieux le nouveau Comité central afin de l'épauler dans ses tâches et de le décharger. En tant

\section{L'année 2012 aura assurément été celle d'une présence politique et médiatique forte et de la reconnaissance confirmée de la FMH comme acteur incontour- nable du monde de la santé.}

que centre de compétence, nous poursuivrons notre action au service de l'ensemble des membres, des sociétés cantonales et spécialisées et des partenaires de la FMH. Mais nous saisirons également l'occasion d'examiner le besoin des membres pour essayer de répondre le mieux possible à leurs attentes et protéger leurs intérêts. Et, ce faisant, nous garderons en mémoire l'intérêt commun de tous, à savoir le bien-être des patientes et des patients.

Que puisse ainsi s'achever l'année 2012, sur une rétrospective critique et positive des mois écoulés et sur une perspective réaliste de l'année à venir. En vous souhaitant à toutes et à tous de pouvoir mener avec sérénité ces réflexions de fin d'année, je joins mes vœux à ceux de tout le Secrétariat général et vous souhaite, à vous et à vos proches, de passer de belles fêtes de fin d'année. 